\title{
Scalp Neoplasm Associated With Cranium Bifidum in a 24-Week Human Fetus
}

\author{
MASON BARR, JR., KATHLEEN P. HEIDELBERGER \\ AND KATERINA DOROVINI-ZIS \\ Departments of Pediatrics (M.B.) and Pathology (K.P.H., K.D.-Z.), University \\ of Michigan, Ann A rbor, Michigan 48109
}

\begin{abstract}
Prenatal ultrasonography at 24 weeks disclosed a fetus with a large mass protruding from the occiput. The mass was an invasive, undifferentiated mesenchymal neoplasm of the scalp associated with a defect in the occipital bone (cranium bifidum). The tumor may have derived from neural crest at the site of rhombencephalic closure. The tumor itself would seem to be a sporadic event, but the cranium bifidum may imply a recurrence risk of 2 $3 \%$ for future pregnancies.
\end{abstract}

A wide variety of congenital tumors have been found in term infants (Bolande, '76). Neoplasms in the neonate are estimated to occur in 1:27,400 live births (Bader and Miller, '79), but many of these display relatively benign behavior (Bolande, '76). With the use of prenatal ultrasonography, sizable tumors have been detected as early as the second trimester (Levi, '79). We report here a 24-week fetus with a prenatally detected large, invasive neoplasm of the scalp associated with a defect in the occipital bone.

\section{CASE REPORT}

A 28-year-old Caucasian gravida-2, abortus-1 woman had an unremarkable pregnancy. She was employed as a dental hygienist but had no exposure to ionizing radiation. She drank one alcoholic drink per month, took acetaminophen infrequently during the implantation period, took prenatal vitamins from the second month of gestation, and did not smoke tobacco, As a teenager and adult she had lived in two Michigan cities in which widespread environmental contamination from chemical manufacturing plants has been suspected. There was no history of neural tube defects or childhood neoplasms among the first-, second-, or third-degree relatives. At 23 weeks gestation, a routine ultrasonogram disclosed a large mass on the posterior aspect of the fetal head. The pregnancy was electively terminated on the 166th postmenstrual day.

\section{GROSS EXAMINATION}

The 564-gm, nonmacerated female fetus was normally grown for the gestational age.
There was a $45 \times 60 \times 35 \mathrm{~mm}(55.5 \mathrm{gm})$ mass over the occiput (Fig. 1a), extending over the left side of the head anterior to the auditory meatus (Fig. 1b). The left pinna was undeveloped but the auditory canal was patent. The right ear and side of the head were normal. The mass was trilobed, with a soft superior portion and two firm lower portions that were separated in the midoccipital line by a membrane-covered fossa. The superior lobe was covered by intact scalp, but the apices of the inferior lobes were eroded, exposing pale tan tissue at the surface. At no point was the mass adherent to the underlying muscle or periosteum.

The tumor was composed of solid tissue intermixed with large and small cystic spaces and hemorrhage. The margins of the superior lobe were distinct, but the margins of the inferior lobes, particularly the left, were quite indistinct and appeared to blend with the adjacent normal subcutaneous tissue.

There was a $10 \times 6 \mathrm{~mm}$ hiatus in the lower portion of the occipital bone (Fig. 1c) but there was no protrusion of intracranial contents out of or extension of tumor into this hole. The skull plates and their contours were normal, although there was a small dura/periosteum-bridged gap in the left lambdoidal suture.

The gross appearance and weight of the brain were normal for the gestational age (Fig. 1d). Elsewhere, there was mild micrognathia but no other malformation or deformation of the craniofacies, neck, thorax, abdomen, genitals, back, or extremities. De-

\footnotetext{
Received August 23, 1985; accepted November 20, 1985.
} 

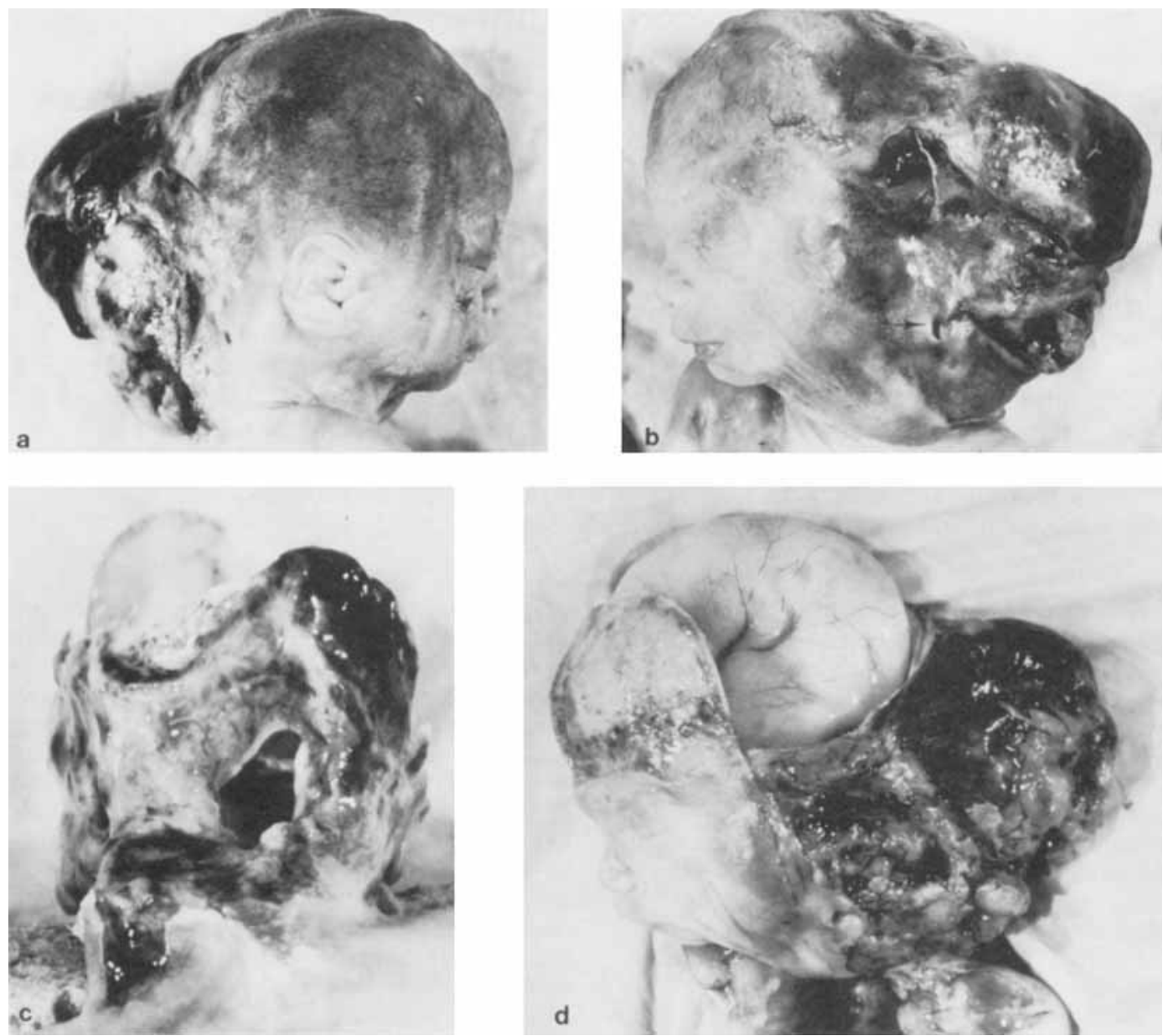

Fig. 1. Malignant neoplasm of scalp. a. Right lateral view of fetus showing normal pinna and a mass protruding from occiput. $b$. Left lateral view showing deformity of soft tissues and anterior extension of mass with failure of development of left pinna (arrow). c. Bony defect in occiput after removal of brain and mass. $d$. Left parietal bone removed, showing fungating mass posteriorly and normal brain.

tailed dissection of the cervical, thoracic, abdominal, and pelvic viscera showed no abnormalities. No metastatic tumor was found.

\section{MICROSCOPIC EXAMINATION}

Multiple sections of the neoplasm were similar in that the grossly noted cysts were less apparent and the mass appeared more generally solid. Lining cells of the cysts were flattened and nonspecific, save for a single focus of ciliated, columnar epithelium. There were areas with suggestions of primitive cartilage formation, but no teratomatous ele- ments were found. The majority of the neoplasm was composed of very primitive malignant cells of two populations. There were larger cells with abundant, eosinophilic cytoplasm and large nuclei with sometimes conspicuous nucleoli and smaller cells with clear cytoplasm and smaller, denser nuclei. In some areas, the two populations were in termingled; in other areas, they aggregated with the larger cells in nests, surrounded by the smaller ones (Fig. 2a). Immunohistochemical stain for S-100 protein was strongly positive in the cytoplasm and nuclei of the large cells; the smaller cells were largely 
nonreactive (Fig. 2b). Glial fibrillary acidic protein and keratin stains were negative.

Ultrastructurally, the tumor cells were closely packed and separated form one another by flocculant and fibrillary material and collagen fibrils. Closely apposed cell processes occasionally displayed rudimentary intercellular junctions (Fig. 2c). The cytoplasm contained variable numbers of intermediate filaments measuring 10-12 nm in diameter, prominent, focally dilated rough endoplasmic reticulum, mitochondria, and rare tubular inclusions (Fig. 2c,d). The nuclear membrane was often invaginated (Fig. 2d). Cytoplasmic tubular inclusions were rarely seen.

\section{DISCUSSION}

This fetus had a primitive and invasive neoplasm of the scalp associated with a sizable defect of the occipital bone. From its gross appearance, the tumor was at first thought to be a teratoma originating in the posterior midline, but this could not be substantiated on multiple sections and stains. The tumor cells were poorly differentiated without any specific histologic or ultrastructural features that would allow classification of the neoplasm. The only distinct expression of the neoplastic cells was their positive staining for S-100 protein. This protein is found in astrocytes, oligodendrocytes and neurons in the central nervous system and in Schwann cells in the peripheral nervous system. It is also present in a number of tumors of neural crest derivation (Stefansson et al., '82), chondrocytic tumors (Nakamura et al., '83), and a few other tumors such as mixed tumors of salivary gland and benign and malignant tumors of breast (Nakajima et al., '82). The location of this tumor, its histologic and ultrastructural features, and its negative staining for glial fibrillary acidic protein and keratin rule out glial neoplasm, meningioma and most of the tumors known to contain S-100 protein. However, we can not entirely rule out partial cartilaginous differentiation, expressed mainly by the larger cells of this otherwise undifferentiated mesenchymal neoplasm.

The S-100 protein staining and the association of the tumor with the skull defect suggest the possibility that the origin of the tumor was neural crest at the margin of rhombencephalic closure. The region of the defect is just rostral to the site of first closure of the neural tube and the adjacent somites give rise to the supraoccipital portion of the occipital bone (Lemire et al., '75). Cephalad from these somites, the "mesodermal" tissues are derived from neural crest (Johnston et al., '77). In this fetus, neural tube closure occurred, as determined by an intact rhombencephalon, but the formation of an epidermal (skin) covering of the neural tube is less sure. It is possible that an early proliferation of cells in the posterior midline could have disturbed the evolution of the skin in this region and also interfered with emplacement of somite-derived tissues, producing the occipital defect. On the other hand, the occipital defect may have been primary, with later malignant transformation of rests of neural crest or other mesencyme.

Another puzzling feature of this case is the virtual aplasia of the left pinna with preservation of the external auditory metus. The pinna forms from surface hillocks on either side of the first branchial cleft. If the neoplasm had extended into the region of the branchial cleft at such an early stage of development as to prevent formation of the ear hillocks, it seems inconceivable that the auditory meatus could have developed normally. On the other hand, if the tumor extension into the region was delayed beyond the time of pinna formation, it seems likely that the formed pinna would be carried, more or less intact, on the surface of the expanding tumor. We can offer no resolution to this apparent paradox.

Perhaps more important than a precise histologic classification of the neoplasm is the need to distinguish it from lesions that may look similar on prenatal ultrasonograms. Cystic hygroma and encephalocele were the main diagnostic considerations in this case and led to the termination of the pregnancy. These two lesions may be isolated occurrences or they may be part of multiple anomaly syndromes with genetic implications. Whether or not cranium bifidum, with or without occipital encephalocele, is part of the neural tube defect spectrum that includes anencephaly and myeloschisis is not established (Lemire et al., '75). The latter malformations are defects of neurulation while in cranium bifidum, as seen in this case, neurulation has been completed. However, because of the uncertainty a conservative estimate of $2-3 \%$ for the recurrence risk, based on a possible multifactorial etiology of cranium bifidum, was proposed by our genetic consultants. The mother was advised to 

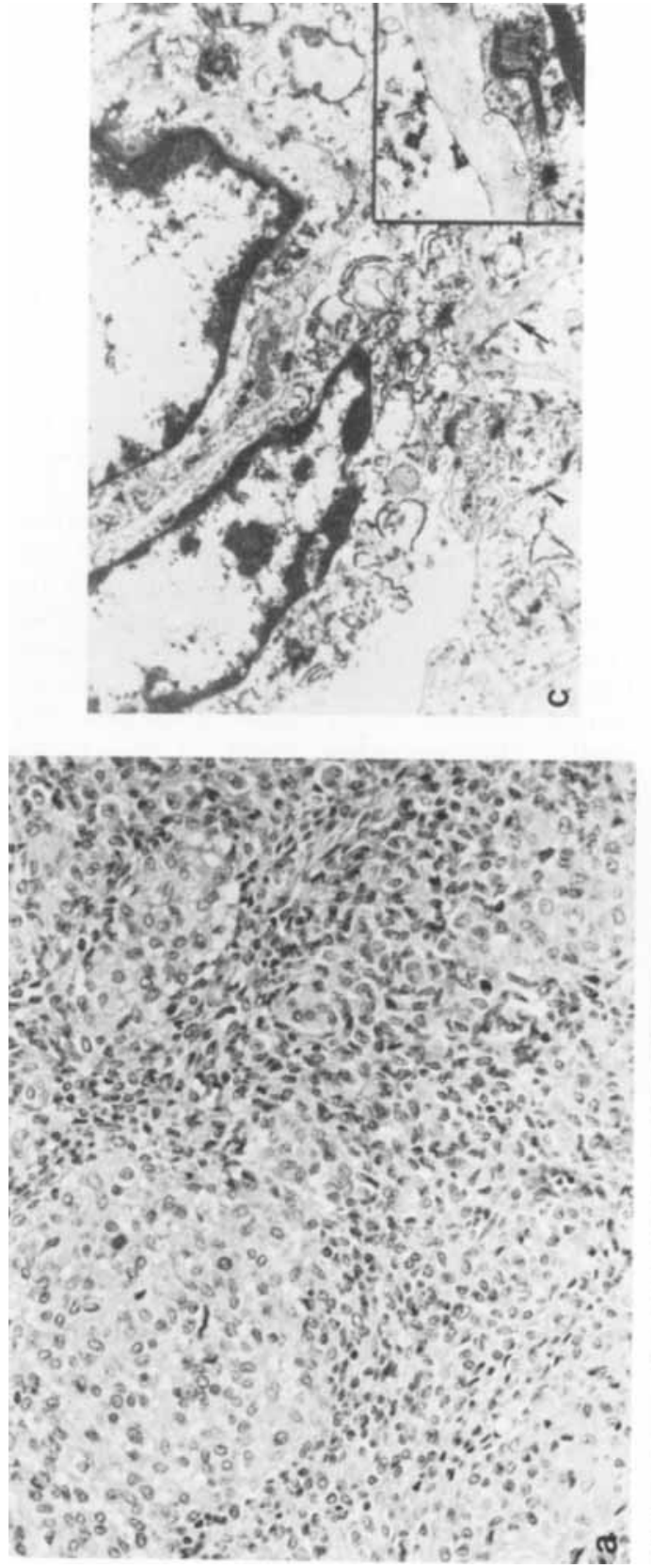
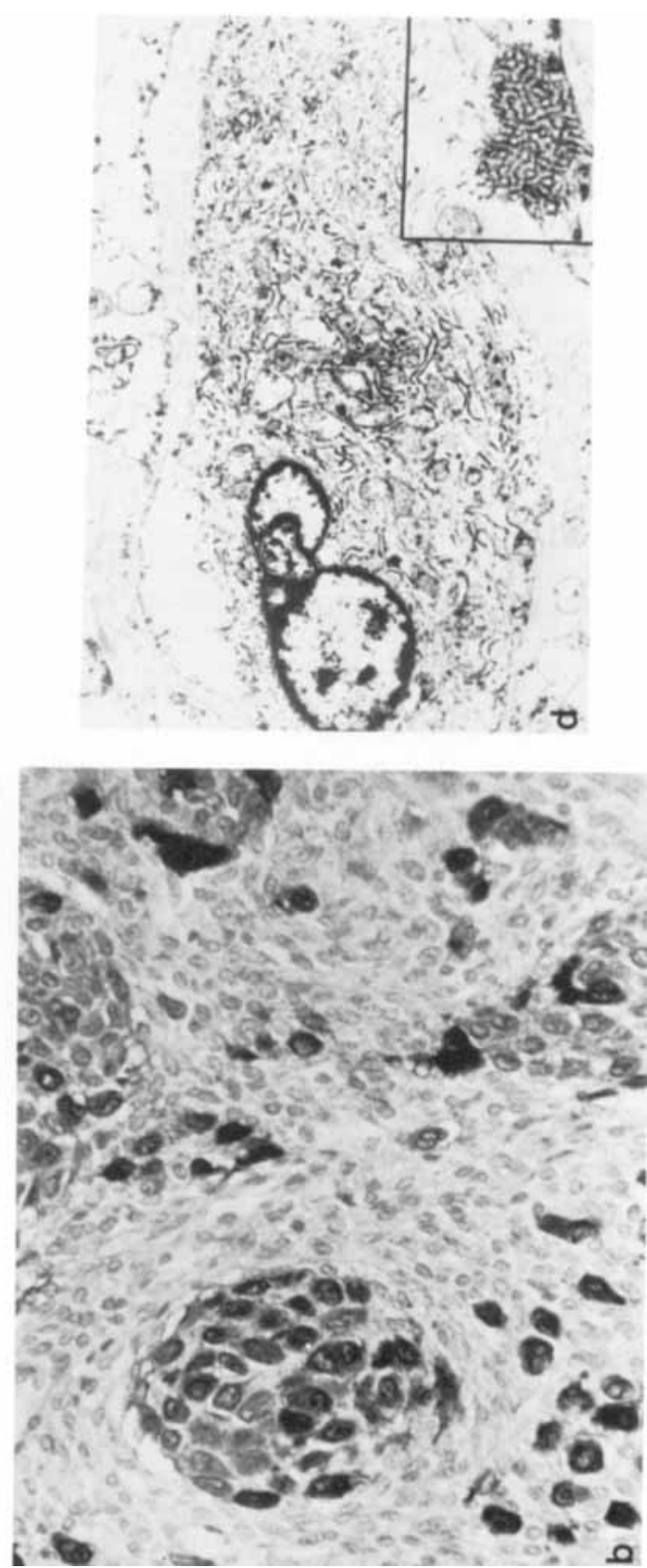
have fetal ultrasonography in future pregnancies with an eye to detecting encephalocele or one of the more common neural tube defects. On an empiric basis, the risk for having another fetus with a scalp tumor would seem to be negligible since this is the only case of this lesion that we have been able to discover.

\section{LITERATURE CITED}

Bader, J.L., and R.W. Miller (1979) U.S. cancer incidence and mortality in the first year of life. Am. J. Dis. Child., 133:157-159.

Bolande, R.P. (1976) Neoplasia of early life and its relationships to teratogenesis. In: Perspectives in Pediatric Pathology, vol 3. H.S. Rosenberg and R.P. Bolande, eds. Year Book Publishers, Chicago, pp. 145-183.

Johnston, M.C., G.M. Morriss, D.C. Kushner, and G.J. Bingle (1977) Abnormal organogenesis of facial struc- tures. In: Handbook of Teratology, vol 2, J.C. Wilson and F.C. Fraser, eds. Plenum Press, New York, pp. 421-451.

Lemire, R.J., J.D. Loeser, R.W. Leech, and E.C. Alvord (1975) Normal and Abnormal Development of the $\mathrm{Hu}$ man Nervous System. Harper and Row, Hagerstown, pp. 289-298.

Levi, S. (1979) Uses of ultrasonography in fetal and perinatal medicine. In: Reviews in Perinatal Medicine, vol 3, E.M. Scarpelli and E.V. Cosmi, eds. Raven Press, New York, pp. 155-195.

Nakajima, T., S. Watanabe, and Y. Sato (1982) An immunoperioxidase study of S-100 protein distribution in normal and neoplastic tissues. Am. J. Surg. Pathol., 6:715-727.

Nakamura, Y., L.E. Becker, and A. Marks (1983) S-100 protein in tumors of cartilage and bone. Cancer, 52:1820-1824.

Stefansson, K., R. Wollmann, and M. Jerkovic (1982) S100 protein in soft-tissue tumors derived from Schwann cells and melanocytes. Am. J. Pathol., 106:261-268.
Fig. 2. Malignant neoplasm of scalp. a. Section of neoplasm with nodules of large, rounder cells separated by strands of smaller, more spindled cells. Mitoses are found in both components $(\mathrm{H} \& \mathrm{E} ; \times 132)$. b. S-100-stained area with larger, positive cells admixed with smaller, negative cells (S-100, 1:1,000 dilution; $\times 208$ ). c. Tumor cells separated by collagen (arrow) are occasionally joined together by rudimentary junctional complexes (arrowhead and inset) $(\times 17,750$; inset $\times 24,800)$, d. Intracytoplasmic fibrils, rough endoplasmic reticulum, and mitochondria in tumor cell with convoluted nucleus $(\times 13,300)$. Inset: cytoplasmic tubular incisions are rare $(\times 42,500)$. 